\title{
DELIMITACIÓN Y ESTUDIO DE CUENCAS HIDROGRÁFICAS CON MODELOS HIDROLÓGICOS
}

\author{
Alejandra M. Geraldi, 2; M. Cintia Piccolo² y Gerardo M. E. Perillo ${ }^{1}$ \\ ${ }^{1}$ Universidad Nacional del Sur (Bahía Blanca, Argentina) \\ ${ }^{2}$ Instituto Argentino de Oceanografía (Bahía Blanca, Argentina)
}

\section{RESUMEN}

La cuenca Las Encadenadas del Oeste se localiza en el sudoeste de la provincia de Buenos Aires. Es una cuenca endorreica y un escenario de suma fragilidad ante eventos hidrológicos extremos, debido a su incapacidad para evacuar grandes volúmenes de agua. Por esta razón, es de suma importancia el conocimiento y la delimitación de su cuenca hidrológica. El objetivo del presente trabajo es establecer la dirección de la escorrentía superficial a partir de la aplicación de modelos hidrológicos e identificar divisorias de aguas. Se aplicó el modelo «Hydrologic Modeling», de Arc Gis, el cual consiste en determinar todas las celdas que drenan a una en particular. Se obtuvo una clasificación de las subcuencas en mayores, medias y conos aluviales activos. Los primeros formados por arroyos principales permanentes, las segundas formadas por arroyos secundarios que nacen de cárcavas y los conos aluviales sin cursos definidos, formados en la desembocadura de los arroyos principales y secundarios.

Palabras clave: hidrografía, modelos hidrográficos, cuenca, SIG, escorrentía.

\section{ABSTRACT}

Las Encadenadas del Oeste basin is located in the southwestern of the Buenos Aires province. It is an endorheic basin and an extremely fragile scenario for its inability to pass large volumes of water. For this reason, it is very important to study and understand the hydrology of its watershed. The aim of this study is to establish the surface runoff direction of the basin using an hydrological 
model and to identify its watershed. The applied Arc Gis model was «Hydrologic Modeling». The model determines all cells that drain in a particular one. A classification of the sub-basins in larger, medium and active alluvial cones was obtained. Larger basins are permanent major rivers, medium ones are non permanent streams and the alluvial conos were the ones formed at the mouths of major and minor streams.

Key words: hydrography, models, basins, GIS, runoff.

\section{INTRODUCCIÓN}

La identificación geomorfológica de una región es de fundamental importancia, puesto que constituye uno de los factores principales que dominan la dinámica hídrica (Lexow, 2002). La morfología regional, condicionada por procesos de sedimentación, erosión, depositación y transporte da lugar a la existencia de cuencas hidrográficas. El agua, a través de su capacidad de erosión y transporte de sedimentos, se comporta como un factor relevante en el modelado de la superficie terrestre y de sus paisajes (Melo, 2003).

La caracterización geomorfológica ambiental de una cuenca fluvial se determina en primera instancia por su conocimiento morfométrico. Este último es uno de los aspectos más influyentes en la ecología acuática, dado que muchos indicadores, composición físico-química, sedimentación dependen de la forma de la cuenca. La morfometría sirve, además, para hallar parámetros que caractericen un ambiente geomorfológico y que sean susceptibles de un tratamiento estadístico que permita disminuir la subjetividad en las conclusiones (Doffo y Bonorino, 2005).

El espectro cronológico de la cuenca Las Encadenadas del Oeste incluye morfologías longevas en los sistemas serranos, anteriores al Cenozoico, hasta otras del Holoceno tardío (Zarate et al., 2005). La cadena serrana, localizada en el sector Sur de la cuenca, tiene forma sigmoidal y se interpreta como resultado de la presencia de componentes de cizalla durante la deformación (Martinez, 2001) (Fig. 1).

Los escurrimientos superficiales son controlados por la pila sedimentaria que cubre el área y que data de los últimos 5 millones de años. Estos sedimentos fueron depositados por el viento y su espesor llega al centenar de metros en la parte superior (González Uriarte y Orioli, 1998). La base de estos sedimentos fueron denominados por Ameghino (1880) con el término de Pampeanos. Cubre esta pila sedimentaria otra identificada también por Ameghino como Postpampeano (González Uriarte y Orioli, 1998). El conocimiento de estos sedimentos es importante, puesto que ejercen un control sobre el paisaje actual y los materiales sobre los que evolucionan los suelos. 
Las cuencas hidrográficas son consideradas sistemas abiertos, que reciben energía y materia de la atmósfera y procesos endogénicos y la pierde a través del caudal y la descarga de sedimentos (Gregory y Walling, 1973). La delimitación de la cuenca y las subcuencas permitirá un mejor cuidado de los recursos hídricos. Las lagunas Encadenadas del Oeste, conformadas por Laguna Epecuén, La Paraguaya, Venado, Del Monte, Cochicó, Alsina e Inchauspe (Fig. 1), se localizan en el centro de la provincia de Buenos Aires. Es una depresión lagunar que separa el piedemonte del Sistema de Ventana de la llanura arenosa del Noroeste.

La cuenca comprende los partidos de Adolfo Alsina, Guaminí, Daireaux, Coronel Suárez; Saavedra y Puán (Fig. 1). La principal actividad es agrícola, afectada por la alternancia de periodos secos y húmedos, producto de un clima templado de transición. Las lagunas del sistema son lagunas de llanura de profundidad media, que no estratifican térmicamente. Todos los aportes encauzados se localizan al Sur de las mismas, sin presencia de ellos por el Norte. Cada laguna tiene su unidad colectora propia, conformada por un arroyo principal que, en la mayoría de los casos, es alimentada por precipitaciones de la zona alta de la cuenca. Los arroyos más importantes son Pigüé (laguna Epecuén), Venado (laguna Venado), Guaminí (laguna Del Monte), Cochicó (laguna Cochicó), Cura Malal, Pescado y Corto (laguna Alsina) (Fig. 1).

Figura 1. Localización del área de estudio

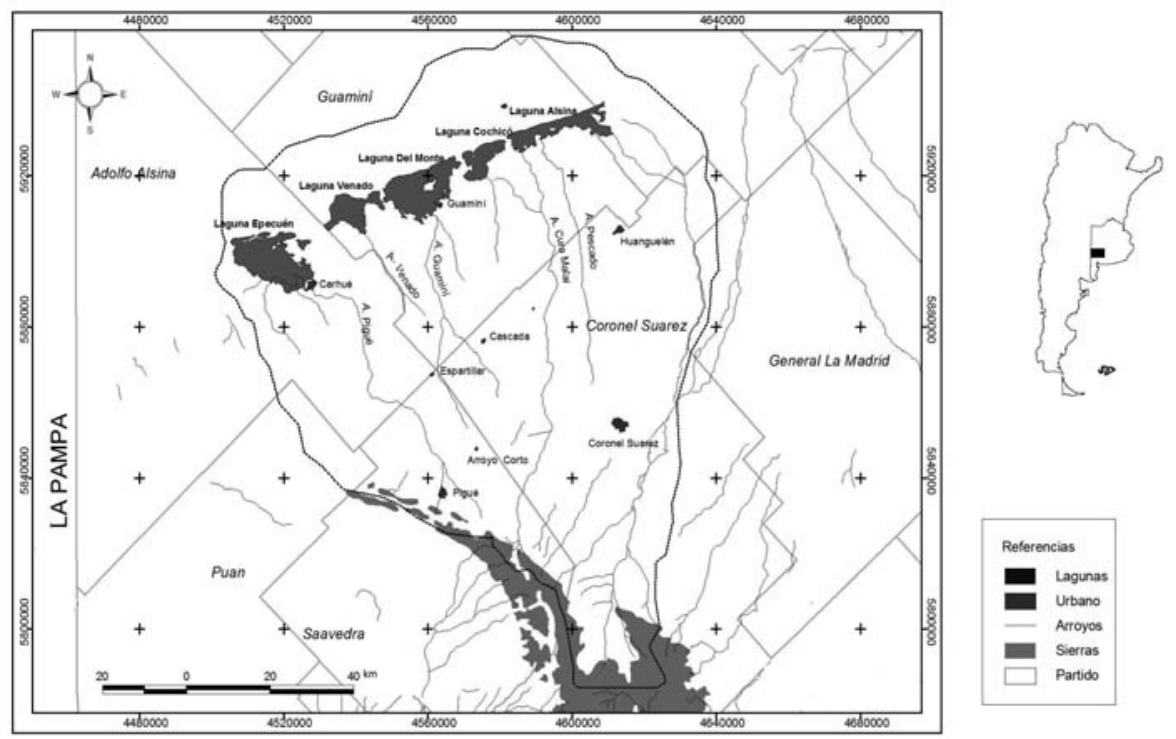

Elaboración propia 
La cuenca de las lagunas es un sistema fluviolacustre de tipo endorreica, comprendiendo unidades morfológicas diferentes. La altura media del área es de 100 msnm, constituyendo la parte más baja del sistema el lago Epecuén (90 msnm), en el cual desembocan todas las aguas de la cuenca (Fig. 1). Cuando la laguna Epecuén alcanza la cota 92,75 m queda seccionada formando otra laguna, llamada Alpataco, nivel alcanzado en 1952 (Montesarchio, 1990). El objetivo del presente trabajo es establecer la dirección de la escorrentía superficial a partir de la aplicación de modelos hidrológicos e identificar divisorias de aguas.

\section{MATERIALES Y MÉTODO DE TRABAJO}

Para analizar la topografía se digitalizaron las curvas de nivel a 2,5 metros de equidistancia, utilizando el material cartográfico del Instituto Geográfico Militar. Las cartas topográficas se georreferenciaron y se generó un mosaico donde se incluyó la totalidad de la cuenca. La red de drenaje se digitalizó de las cartas topográficas y se la actualizó con imágenes satelitales Landsat 5 TM proporcionadas por la CONAE (Comisión Nacional de Actividades Espaciales).

Para realizar el estudio del relieve se efectuó un Modelo Digital del Terreno (DEM), creando una Red de Triangulación Irregular (TIN) con los datos de las curvas de nivel. Un TIN representa una estructura de datos vectoriales utilizada para almacenar y desplegar un modelo de superficie. Este divide una superficie topográfica en un grupo de triángulos contiguos no superpuestos. Los puntos de muestras forman vértices triangulares o nodos y los valores de elevación de estos nodos son interpolados para crear una superficie continua. Los triángulos son construidos de acuerdo con una técnica matemática denominada Triangulación Delaunay, la cual garantiza que un círculo dibujado a través de nodos de cualquier triángulo no contendrá ningún otro punto ingresado (Sione et al., 2004).

Se aplicó el modelo «Hydrologic Modeling» (HM) de Arc Gis, que determinó las direcciones de las corrientes. Este modelo trabaja con la topografía de la cuenca, simulando escurrimientos superficiales. Consiste en determinar todas las celdas que drenan a una en particular. Se evalúa el recorrido del flujo para cada celda a partir de la malla de direcciones de flujo. De esta manera, aquellas celdas que tengan mayor acumulación del flujo serán las que forman los canales de drenaje.

La gran acción antrópica que presenta el área ha modificado muchos cursos de agua y divisorias de cuenca por medio de la construcción de canales, ferrocarriles y compuertas. Se hizo necesario, entonces, un trabajo de campo para verificar divisorias de agua y mediciones con Global Position System (GPS), controlando, además, altitud y coordenadas geográficas. 
Una vez obtenidos todos los datos (curvas de nivel, puntos GPS, divisorias de aguas antrópicas, canales, escurrimientos), se delimitó la cuenca y luego las subcuencas para cada uno de los arroyos. La delimitación se comenzó por la desembocadura, siguiendo los puntos más elevados hasta cerrar el circuito. Se utilizó el software Arc Gis 9, con sus respectivas extensiones. Este programa permitió obtener de forma inmediata parámetros de la topografía, longitud de los cauces, superficie de la cuenca y subcuencas.

\section{RESULTADOS}

El Modelo Digital de Elevaciones (Fig. 2) demuestra la singularidad del relieve de la cuenca. Las alturas en el área están comprendidas desde los 82 msnm (altura mínima), que corresponde a la cota mínima de la laguna Epecuén en el Oeste, y 1015 msnm (altura máxima), correspondiente a la cota en la que nacen los arroyos que conforman las cuencas mayores en el sistema Ventania. La diferencia entre estas dos alturas extremas definen un relieve que sugiere alturas y pendientes significativas favorables a la génesis y activación de procesos erosivos y de transporte de materiales. Se observa que las alturas mayores se localizan al Sur de la cuenca y las menores al Norte de la misma. El resto del paisaje se presenta debajo de la cota de $400 \mathrm{~m}$. Con la delimitación de la cuenca se calculó que la extensión de la misma es de 1115248 ha, incluyendo arroyos, lagunas y relieves diferentes.

Figura 2. Modelo Digital de Elevación de las lagunas las Encadenadas del Oeste teniendo como base las curvas de nivel a 2,5 m de equidistancia

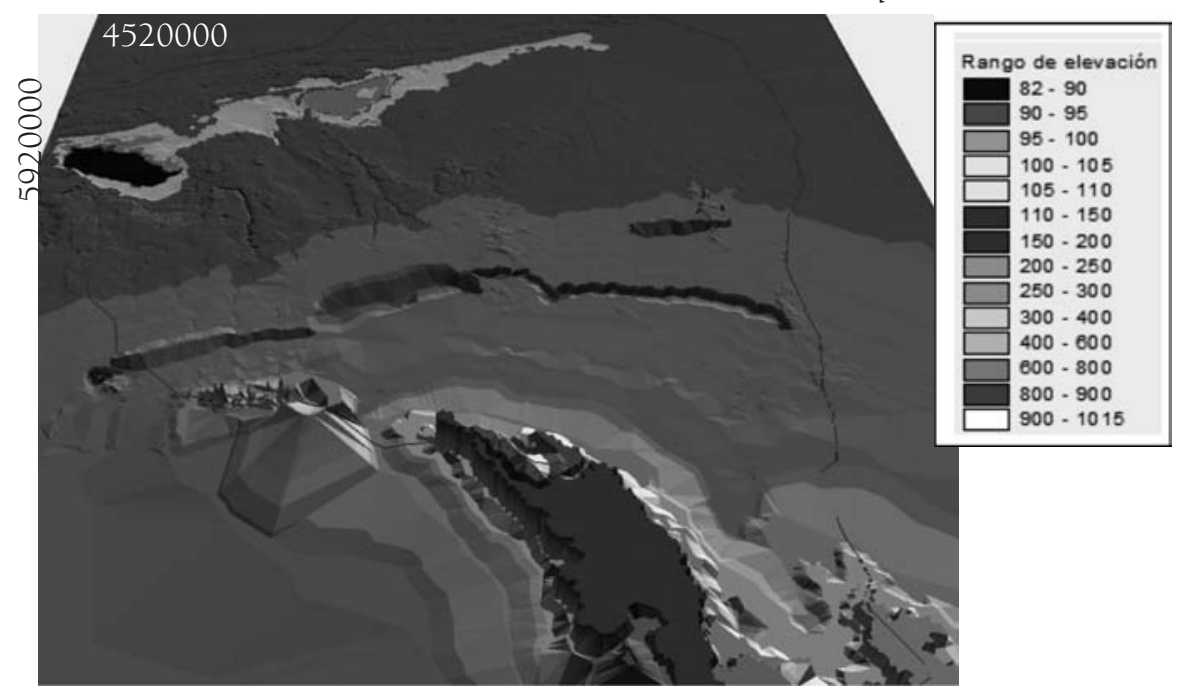

Investigaciones geográficas, $n^{\circ}$ 52, pp. 215 - 225 
La aplicación del modelo «Hydrologic Modeling» permitió ver la dirección de los escurrimientos hasta encausarse en un curso de agua principal (Fig. 3). Dichos escurrimientos siguieron el comportamiento de la topografía regional (Fig. 4). Así, estos definen los afluentes de los arroyos y, a partir de aquí, se precisa hacia dónde drenan las aguas.

Figura 3. Escurrimientos superficiales en la cuenca Las Encadenadas del Oeste calculadas a partir de la aplicación del modelo «Hydrologic Modeling»
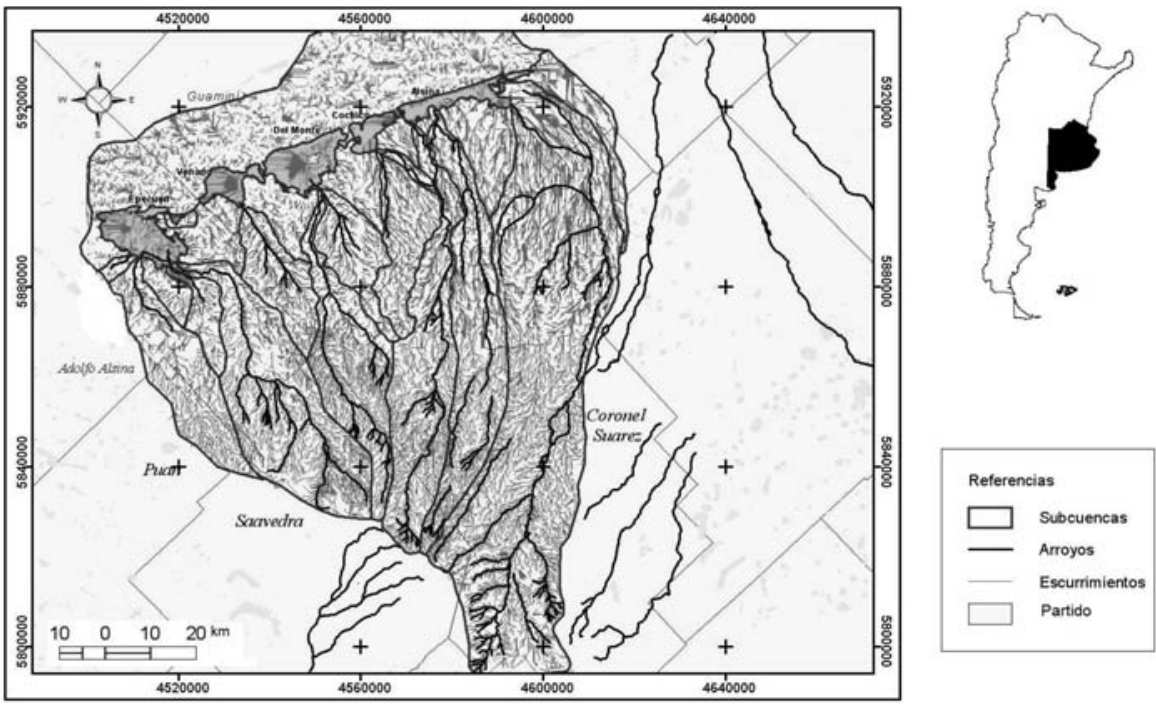

Figura 4. Escurrimientos superficiales sobre el MDE en la cuenca Las Encadenadas del Oeste

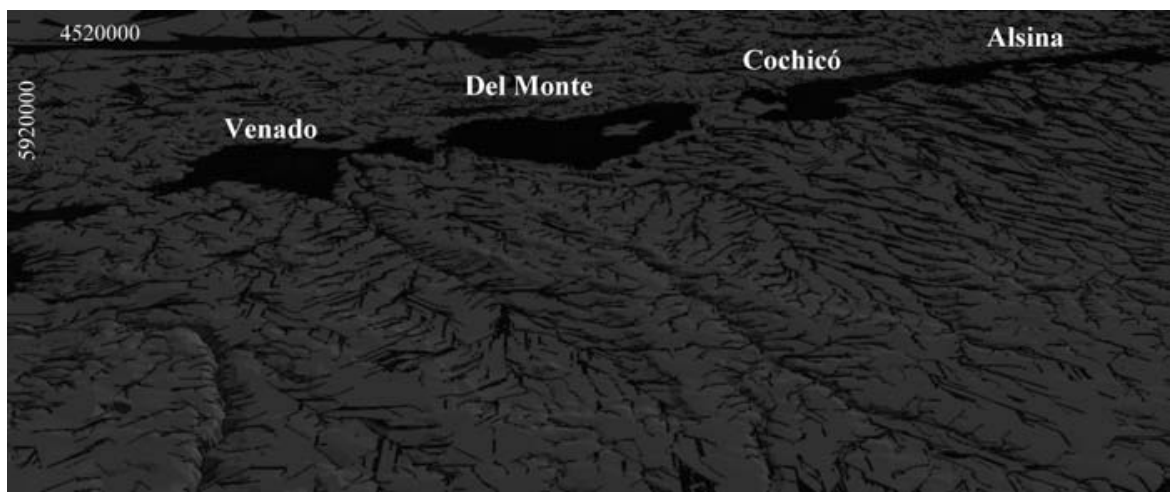


Los escurrimientos están bien definidos hacia los arroyos principales y sus afluentes (Fig. 4). Sin embargo, el sector localizado al Norte de las lagunas presenta escurrimientos difusos que no definen cauces de ningún tipo, debido a que el sector es una zona de médanos. En esta área, durante los periodos húmedos, se forman lagunas de carácter temporario, mientras que durante los periodos secos son cubiertas por pastizales.

Con los cursos definidos y el MDE se delimitaron las subcuencas de cada uno de los arroyos que conforman la cuenca Encadenadas del Oeste. Las mismas quedaron clasificadas de la siguiente manera:

a. Subcuencas Mayores: La integran aquellas que desarrollan su superficie hasta la zona de las Sierras (Fig. 5); contienen los arroyos principales de la cuenca. Estas subcuencas son:

1. Subcuenca Arroyo Corto (CAC)

2. Subcuenca Arroyo Pescado (CP)

3. Subcuenca Arroyo Cura Malal (CACM)

4. Subcuenca Arroyo Guaminí (CAG)

5. Subcuenca Arroyo Pigüé (CAP)

b. Subcuencas Medias: Nacen en la mitad de la cuenca, en la zona caracterizada como llanura (Fig. 5). Se localizan intercaladas con las anteriores y la constituyen aquellos arroyos menores que son de carácter intermitente y cuyos nacientes no se localizan en las Sierras. Los arroyos nacen de cárcavas. Las mismas son:

1. Subcuenca Arroyo Pull (CPP)

2. Subcuenca Arroyo Venado (CAV)

3. Subcuenca Arroyo Malleo Leufú (CAML)

4. Subcuenca Arroyo Cochicó (CC)

c. Conos Aluviales activos: Son cuerpos sedimentarios en forma triangular, su base ancha en contacto con las lagunas y el vértice es la zona de contacto con las subcuencas. Los abanicos aluviales se forman en la desembocadura de los ríos, debido a la reducción de la pendiente, con disminución de su velocidad y el ensanchamiento del valle, constituyéndose en el lugar de depositación de los materiales erosionados en los sectores superiores (Strahler, 1952). Tienen forma plana y están formados por materiales de depósitos fluviales. Los escurrimientos tienen un comportamiento anárquico. Estas unidades se localizan entre las lagunas y las diferentes subcuencas (Fig. 5), es por ello que se los denominó en este trabajo de la siguiente manera:

1. Cono Aluvial del lago Epecuén (CE)

2. Cono Aluvial Epecuén - Venado (CEV)

3. Cono Aluvial Venado Del Monte (CVM) 

4. Cono Aluvial Del Monte- Cochicó (CMC)
5. Cono Aluvial Cochicó- Alsina (CA)
6. Cono Aluvial Cura Malal- Pescado (CCP)
7. Cono Aluvial Pescado - Corto (CPC)

d. Subcuenca Medanosa (CN): Se localiza al Norte de las lagunas y se caracteriza por la presencia de médanos. No tiene aportes fluviales de ningún tipo. Se han identificado médanos, mantos de arena, depresiones intermedanosas y cubetas de deflación. Se han descrito cuatro unidades litoestratigráficas y cuatro paleosuelos (Dillon et al., 1985). Los limos compactos de la Formación Epecuén han sido reconocidos a diferentes profundidades (Dillon et al., 1985). Las arenas finas a limosas de la Formación Hereford han sido atribuidas al Pleistoceno superior. Sobre ellas se han reconocido los limos arcillo-arenosos a limos-arcillosos de la Formación Carlos Tejedor. Posiblemente se correspondan con los extendidos y característicos niveles de origen ácueo «lujanenses» que caracterizan el Pleistoceno final en la provincia de Buenos Aires. Finaliza la secuencia con la depositación de arenas finas eólicas de la Formación Las Lilas (Holoceno) (Isla et al., 2003).

Se observa en la figura 5 que, en la subcuenca del arroyo Corto, la divisoria atraviesa el curso de agua. Esto se debe a que es de origen puramente antropogénico; en ese punto se ha realizado un taponamiento del arroyo, desviando los caudales hacia cuencas diferentes.

Figura 5. Identificación de las subcuencas y división de las mismas en mayores, medias, conos aluviales y medanosa que forman la cuenca Las Encadenadas del

Oeste según los modelos MDE, HM y al trabajo de campo
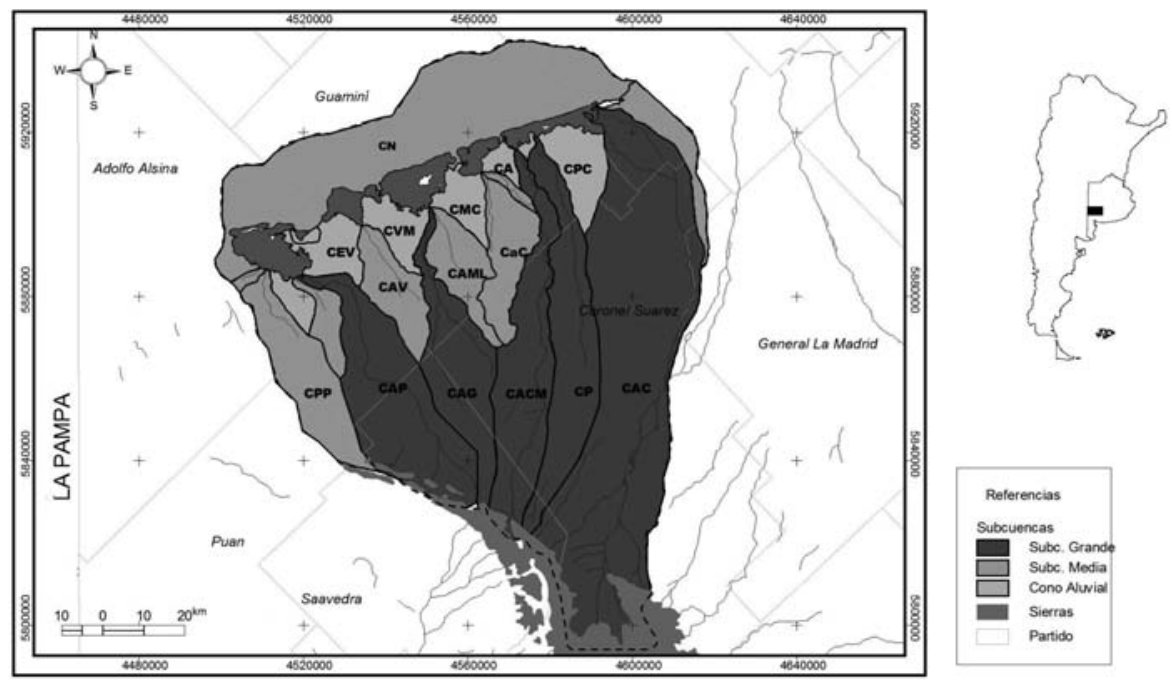
Las cuencas hidrográficas son zonas de articulación entre los habitantes que la forman, sobre todo por el uso del recurso. Sin embargo, en muchos casos, las divisiones de cuencas no coinciden con las divisiones políticas y la planificación no resulta armónica. En la cuenca de las Encadenadas ocurre esto, con el agravante de que intervienen varios municipios que hacen uso del recurso, ignorando que todos pertenecen al mismo sistema. En las subcuencas analizadas, la cuenca del arroyo Corto es la que mayor superficie presenta y, en segundo lugar, la del arroyo Pigüé (Tabla 1). Estas cuencas son, junto con la del arroyo Cura Malal, las que presentan los arroyos de mayor longitud (Tabla 1). Por otra parte estas son las que tienen una topografía compleja, en la cual la cabecera de los arroyos se localiza a más de 800 msnm y en su cuenca media y desembocadura transcurren en un relieve muy plano.

En cuanto a la longitud de los cauces principales, el arroyo de mayor longitud es el Arroyo Corto (Tabla 1), que, además, presenta la mayor longitud total de cauces $(533 \mathrm{~km})$. Los arroyos de menor longitud son el Malleo Leufú (16,6 $\mathrm{km}$ ) y el Cochicó $(20,14 \mathrm{~km})$. La menor longitud total de cauces es la del arroyo Cochicó, que no presenta afluentes en todo su recorrido.

Se define el tiempo de concentración (TC) como el tiempo que demora una partícula de agua en viajar desde el punto más remoto hasta el punto de interés. Corresponde al lapso entre el final de la lluvia y el momento en que cesa el escurrimiento superficial (Fuentes Junco, 2004). Los tiempos de concentración en las Encadenadas son largos en las cuencas más redondeadas (Pigüé, Venado y Cochicó), con valores de 35, 36 y 40 minutos, respectivamente.

Tabla 1. Superficie $\left(\mathrm{km}^{2}\right)$, longitud de los arroyos principales (Lc), longitud total (Lt) de los cauces de las subcuencas analizadas y el tiempo de concentración (TC)

\begin{tabular}{|l|r|r|r|r|c|}
\hline Subcuenca & ÁREA $\left(\mathbf{k m}^{2}\right)$ & Lc $(\mathbf{k m})$ & Lt $(\mathbf{k m})$ & TC & Forma \\
\hline Malleo Leufú & 270,00 & 16,60 & 32,06 & 19 & Oblonga \\
\hline Venado & 293,03 & 23,04 & 23,00 & 36 & Ovalada \\
\hline Cochicó & 333,97 & 20,14 & 20,14 & 40 & Oblonga \\
\hline Guaminí & 605,12 & 50,70 & 156,21 & 19 & Alargada \\
\hline Pescado & 791,91 & 70,22 & 180,40 & 15 & Alargada \\
\hline Cura Malal & 829,63 & 106,38 & 168,77 & 17 & Alargada \\
\hline Pigüé & 998,56 & 80,18 & 80,18 & 35 & Alargada \\
\hline Corto & 2893,62 & 160,37 & 533,00 & 17 & Alargada \\
\hline
\end{tabular}

Los máximos de crecidas por inundación son entonces menores para las subcuencas más alargadas y las mayores crecidas se darán en las subcuencas Pigüé, Venado y Cochicó. Se destaca entonces el riesgo de las poblaciones que 
se asientan sobre las llanuras aluviales de los arroyos, las planicies marginales y lagunas. Este es el caso de la localidad de Carhué, que es un espacio de riesgo hídrico importante, por estar en la llanura aluvial y desembocadura del arroyo Pigüé. La misma forma parte de una subcuenca con tiempos largos de concentración, formas redondeadas y, por lo tanto, crecidas más importantes. La colmatación de sectores bajos con capacidad de almacenaje disminuida, como es este caso, se corresponde con anegamientos en áreas naturalmente arreicas.

\section{CONCLUSIONES}

A partir de este análisis surge, por primera vez en el área, una clasificación de las subcuencas que servirá para futuros estudios hidrológicos y para planes de manejo del agua en la zona. Los resultados constataron las divisorias de las subcuencas, que, en algunos casos, son de origen puramente antropogénico, como en el caso de la subcuenca del arroyo Corto. Los escurrimientos siguen el comportamiento de la topografía y sólo en los conos aluviales activos y la subcuenca Norte tienen un comportamiento más anárquico.

El análisis de la morfometría, la topografía y los escurrimientos superficiales advierten que la cuenca es una zona compleja, con dificultades de evacuar volúmenes importantes de agua debido a su topografía. La localidad de Carhué es una de las zonas de mayor riesgo debido a su localización.

El territorio de las cuencas facilita la relación entre sus habitantes, independientemente de que si estos, por razones administrativas, se agrupan en municipios. Todos los que componen la cuenca son dependientes de un sistema hídrico común. Es recomendable, entonces, planes de manejo del agua integrando primero los municipios de las subcuencas y luego todos los municipios que integran la cuenca las Encadenadas. La incorporación a la evaluación del sistema hídrico, de abordajes integrados y el apoyo de herramientas como la modelación permite identificar los cambios inducidos por acciones del hombre, sus efectos y la posible modificación de la calidad ambiental.

\section{REFERENCIAS}

Dillon, A. A.; Hurtado, M. A.; Jiménez, J. E. y Castillo, R. J. (1985): «Consideraciones geomorfológicas y estratigráficas como base del carteo de suelos en un sector de la Pampa Arenosa (Pcia. de Buenos Aires)», en las. Jornadas Geológicas Bonaerenses, Actas, Tandil, pp. 737-749.

Doffo, N. y Bonorino, G. G. (2005): «Caracterización morfométrica de la cuenca alta del arroyo Las Lajas, Córdoba: Un análisis estadístico», Revista de la Asociación Geológica Argentina, vol. 60, n 1, pp. 16-22. 
González Uriarte, M. y Orioli, G. (1998): Carta Geoambiental del partido de Guaminí. Provincia de Buenos Aires. Editorial UNS. Argentina, 243 pp.

Gregory, K. y Walling D. (1973): Drainage basin form and process: A geomorphological approach. Edgard Arnold. Londres-Inglaterra, 45 pp.

isla, F. I.; Ruiz Barlett, E.; Márquez, J. y Urrutia, A. (2003): «Efectos Enso En la Transición entre el espinal y la pradera cultivada en la diagonal Sudamericana, Argentina Central», Rev. CEG, n 17 (1-2), pp. 63-74.

LEXow, C. (2002): Hidrodinámica de la zona no saturada aplicada al estudio del Balance Hidrológico y de contaminantes de los recursos hídricos subterráneos (Cuenca del Arroyo del Águila). Tesis Doctoral en Geología. Universidad Nacional del Sur. Bahía Blanca. Argentina, pp. 175.

Martínez, J. S. (2001): «The geology of Ventania (Buenos Aires Province, Argentina)», Journal of Iberian Geology, n 27, pp. 43-69.

Melo, W. D. (2003): Génesis del Estuario de Bahía Blanca: Relación Morfodinámica y Temporal con su Cuenca Hidrográfica. Tesis Doctoral. Bahía Blanca. Argentina, pp. 202.

Montesarchio, L. A. (1990): «Lagunas Encadenadas del Sudoeste de la Provincia de Buenos Aires: Descripción y análisis de sus características hidrográficas» Revista Universitaria de Geografía, Argentina, n 4 (1 y 2), pp. 159-178.

Sione, W.; Cuello A. y Angelini, M. (2004): Análisis Espacial, Técnicas cuantitativas aplicadas. Curso 9. Especialización en Teledetección y Sig. Editorial de la Universidad Nacional de Luján, pp. 69.

Strahler, A. (1952): «Hypsometric (area-altitud) analysis of erosional relief», Bulletin of Geological Society of America, n 63, pp. 1117-1142.

Zárate M.; Neme, G. y Gil, A. (2005): «Mid Holocene Pleoenvironments and Humna Ocupationin South America», Quaternary International, n 132, pp. 1-3. 